\title{
Alívio de tensões por vibrações sub- ressonantes: análise e parametrização
}

\section{Stress relief by sub-harmonic vibrations: analysis and parametrization}

\author{
Carlos Augusto Pereira Martins ${ }^{1}$, José Carlos Morilla ${ }^{2}$, \\ Paulo Villani Marques ${ }^{3}$ Sérgio Delijaicov ${ }^{4}$
}

\author{
${ }^{1}$ Engebasa - Mecânica e Usinagem Ltda., Cubatão, SP \\ e-mail: programacao@engebasa.com.br \\ ${ }^{2}$ Universidade Santa Cecília, Santos, SP; ${ }^{3}$ UFMG, DEMEC, Belo Horizonte, MG; ${ }^{4}$ Centro Universitário da FEI, S.B do \\ Campo, SP \\ e-mail:morilla@unisanta.br,pvmarques@terra.com.br, sergiode@fei.edu.br
}

\section{RESUMO}

A indústria mundial utiliza a técnica de alívio de tensões por vibrações sub-harmônicas, ou sub-ressonantes, há mais de vinte anos. No Brasil esta técnica é utilizada há cerca de quinze anos, com resultados comprovados por clientes, através de serviços específicos. Nos últimos anos, com a busca por processos alternativos, que visam a economia de energia com consequente redução do aquecimento global, esta técnica pode tornarse muito mais atrativa do que as que usam processos térmicos. Além disso, a redução do tempo do alívio de tensões por vibração em relação ao processo térmico é em torno de $80 \%$, possibilitando maior agilidade na recuperação de peças, fator primordial no atendimento em paradas para manutenção com prazos exíguos.

Este trabalho tem por objetivo comparar o alívio de tensões obtido pelo processo de vibrações subharmônicas com o conseguido pelo tratamento térmico convencional e parametrizar os resultados obtidos na redução das tensões com a diminuição da frequência do pico de ressonância, antes e após o alívio. Este trabalho mostra de forma geral que o alívio de tensões por vibrações sub-ressonantes na profundidade de $0,2 \mathrm{~mm}$ promove alívio de tensões percentuais médios em média $10 \%$ maiores, quando comparados com os resultados obtidos através do tratamento térmico.

Palavras-chave: tensões residuais, alívio de tensões, vibrações.

\section{ABSTRACT}

The worldwide industries use the sub-resonant vibration stress relief for over twenty years. In Brazil, this technic has been used for about fifteen years. In the last years, with the searching of new alternatives process aiming energy economy with consequently the Global Heating reduce, the use of this technique can be very interesting. Moreover, the time reduction between vibration and thermal stress relief processes is around $80 \%$, allowing a faster repairing pieces, which is prime factor to make a maintenance stop in a short time. Meanwhile, the scientific literature is rare about the use and the efficacy of this system.

This work has the propose of comparing the results of sub-harmonic stress relief process with conventional thermal process and correlate them to the decrease of the resonance peak frequencies, comparing before and after treatments. This work introduces some examples of successful works made at industry and presents an experimental research to measure the residual stress, before and after the treatment, comparing to the conventional heat treatment results. This work shows generally the stress relief by sub-resonant vibrations in the $0.2 \mathrm{~mm}$ depth, promotes stress relief on average $10 \%$ higher when compared with the results obtained by the conventional heat treatment.

Keywords: residual stress, stress relief, vibrations.

\section{INTRODUÇÃO}

É comum o aparecimento de tensões mecânicas nos metais em geral, ao sofrerem algum tipo de processo fabril que envolva variação de temperatura, altos gradientes térmicos ou aquecimento e resfriamento não ho- 
mogêneo, em que a peça não possa se dilatar e contrair livremente durante o ciclo térmico como a têmpera e a soldagem. O surgimento dessas tensões também é comum em processos de fabricação que produzam deformação plástica, tais como a usinagem e trabalho a frio ou transformações microestruturais não homogêneas que impliquem em alterações volumétricas, como a transformação martensítica, por exemplo. Tensões residuais são aquelas que permanecem na peça quando todas as suas solicitações externas são removidas. Essas tensões podem resultar em distorções de forma e dimensionais. [1,2,3]. Este problema é muito conhecido nas indústrias metalúrgicas e metal-mecânicas e para minimizar ou eliminar tais distorções, normalmente executa-se após o processo fabril, um tratamento térmico de alívio de tensões, que geralmente envolve grande consumo de energia, em virtude da temperatura e do tempo necessários, tornando-se um processo relativamente lento e oneroso. [4]

Uma alternativa ao alívio térmico de tensões, que vem sendo utilizada já há algum tempo, em várias partes do mundo é a tecnologia que utiliza a vibração mecânica, com esse mesmo objetivo. Neste procedimento, a economia em tempo é cerca de $98 \%$ e em custos energéticos $90 \%$ [4].

O presente trabalho apresenta uma comparação entre o alívio de tensões obtido pelo processo de vibrações sub-harmônicas com o conseguido pelo tratamento térmico convencional. Para isso foram analisados os resultados de medição das tensões residuais em corpos de prova pelos métodos de difração de raios-X e de furo cego. Também foi verificada a correlação entre as tensões residuais e as diferenças da frequência do pico de ressonância dos corpos de prova, antes e após o alívio de tensões por vibrações, e proposto um modelo matemático para a correlação entre a variação da frequência de ressonância e a diminuição das tensões residuais.

\subsection{Tratamento por Vibração Sub-Harmônica}

A vibração sub-harmônica é a que ocorre no trecho compreendido entre o início do aumento da amplitude até o pico de ressonância em uma determinada curva de ressonância, como mostra a Figura 1. [4]

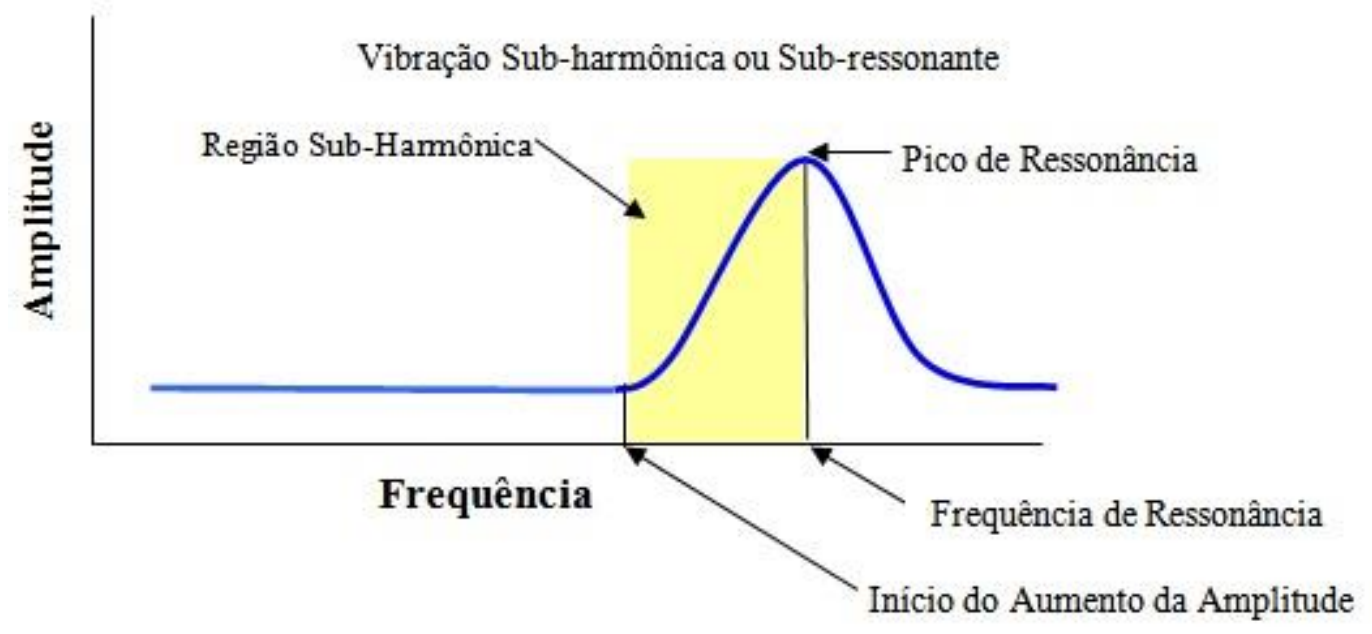

Figura 1: Vibração Sub-harmônica ou Sub-ressonante

O estudo teórico mais aprofundado desta tecnologia se iniciou em 1987 com o trabalho de Richard Skinner [5], na Lockheed Missiles and Aerospace. Skinner buscava o desenvolvimento da vibração como forma de alívio de tensões em peças de aço, alumínio e titânio. Neste estudo foi avaliado detalhadamente, como ocorre a dissipação da energia em função da frequência aplicada. A energia dissipada é entendida como a quantidade de tensões internas contidas na peça que pode ser removida. A região sub-harmônica utilizada nesta tecnologia é a que possui maior capacidade de atuação neste sentido. Pode ser observado na Figura 2, que quanto maior a área formada entre as curvas de carga e sua respectiva histeresis ${ }^{1}$, maior é a quantidade de energia dissipada. Com este estudo, Skinner verificou e comprovou matematicamente, que a energia da vibração Sub-Harmônica possuía a condição mais eficaz para absorção, redução e eliminação das tensões térmicas e mecânicas. Além disso, o estudo de Skinner confirmou que quando se aplica a frequência de ressonância no intuito de remover as tensões internas de uma peça, seu resultado é praticamente nulo, se comparado à região sub-harmônica. Esta comparação pode ser observada no gráfico da Figura 2, através das áreas em

\footnotetext{
${ }^{1}$ Curvas de histeresis - Curva de dissipação de energia mecânica.
} 
"fml" e "frp".

\section{Comportamento da ressonância/não ressonância}

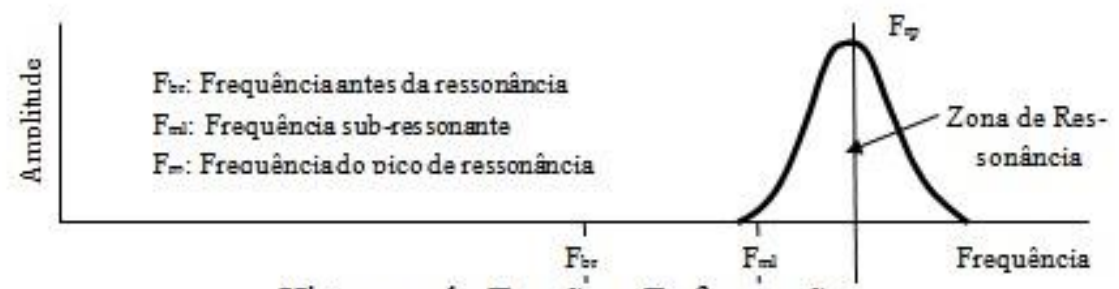

Histerese de Tensã่o e Deformação

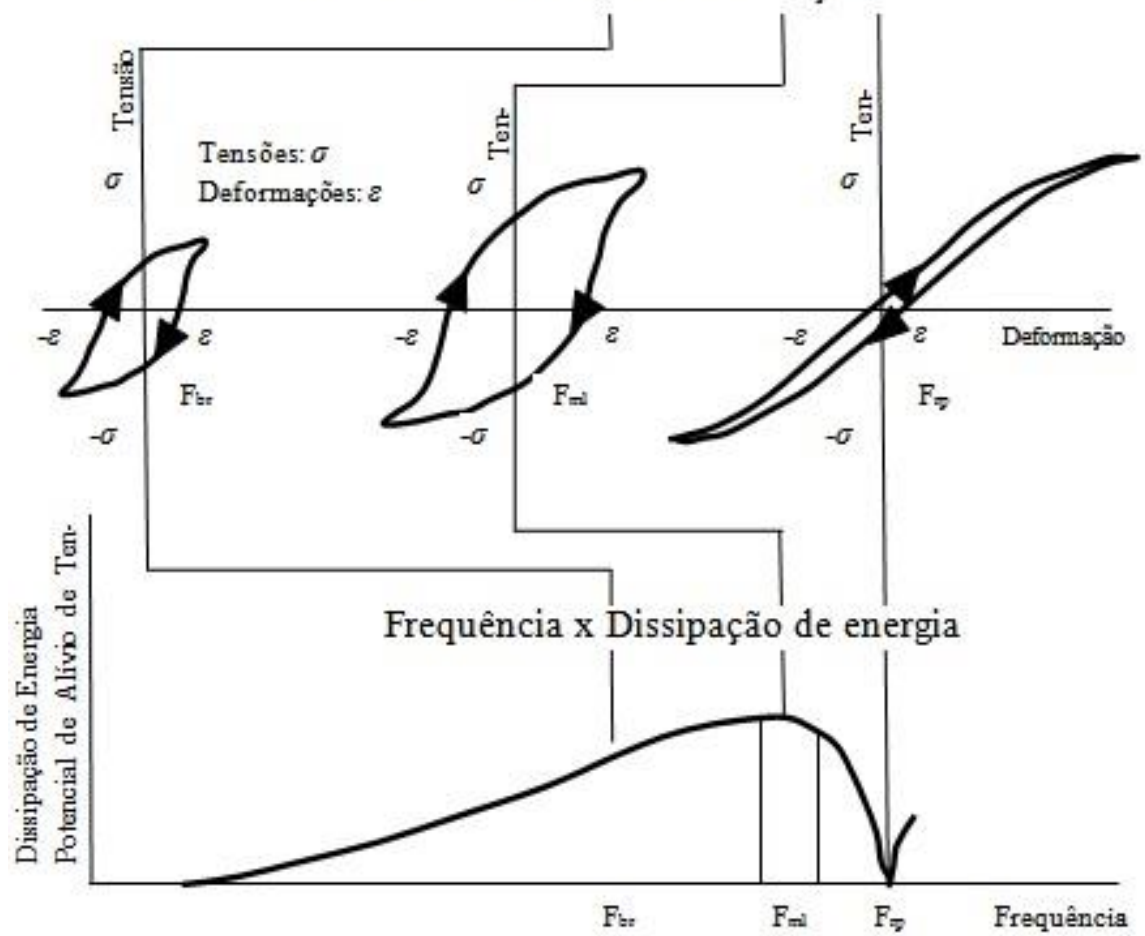

Figura 2: Estudo do efeito das vibrações na dissipação de energia [3].

Em 1987, o estudo "Ultrasonic Evaluation of the Non-linearity of Metals from a Design Perspective" de Wong e Johnson na Universidade de Berkeley - Califórnia [6] demonstrou as características da frequência ressonante ou harmônica dos sistemas metálicos. Os autores mostraram que a frequência de ressonância natural se altera com a presença de tensões residuais. Demonstraram ainda com este estudo que quanto maiores as tensões internas contidas em peças soldadas, maior será a frequência do pico de ressonância.

\subsection{Aplicação do Alívio de Tensões por Vibrações}

Inicialmente determina-se a curva de ressonância da peça a ser tratada (curva 1), calcula-se e aplica-se a frequência que produza uma amplitude de vibração igual a 1/3 da amplitude de ressonância. Terminado o alívio, traça-se uma nova curva de ressonância da peça (curva 2), que é comparada com a anterior. Uma diferença na frequência dos picos de ressonância inferior a $1 \mathrm{~Hz}$ indica que as tensões residuais da peça foram removidas. Caso contrário, uma nova frequência de tratamento é calculada e nova vibração é induzida na peça, repetindo o processo se necessário (curva 3).

A Figura 3 mostra o equipamento instalado numa peça. Maiores detalhes sobre as operações do processo são descritos por MARTINS [7]. 


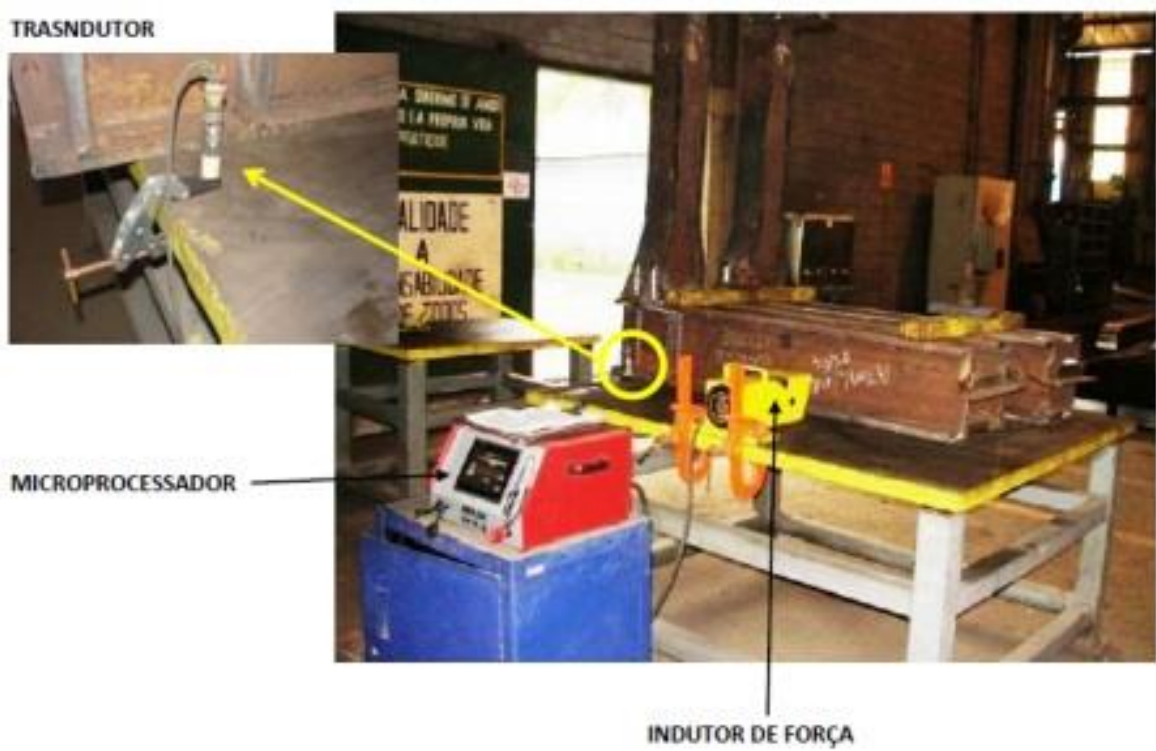

Figura 3: Equipamento de alívio de tensões instalado.

\subsection{Alívio Durante a Introdução das Tensões (Soldagem)}

A aplicação do mesmo processo de alívio de tensões durante a soldagem é conhecida como "condicionamento da solda". Primeiramente é determinada a curva de ressonância da peça a ser soldada, identificando-se a frequência ideal para aliviar as tensões e esta é aplicada durante a soldagem. Desse modo, as tensões são eliminadas logo após seu surgimento, devido à solidificação e resfriamento da solda, minimizando a ocorrência de trincas e de distorções.

O uso desta técnica permite ainda o aumento da corrente elétrica e da velocidade de soldagem, ou obtenção de uma maior penetração, de uma estrutura com grãos mais uniformes e acabamento mais fino, melhorando assim as propriedades mecânicas e aumentando a resistência à fadiga. A ductilidade da solda aumenta em até $400 \%$ em relação a uma solda não condicionada [8]. Neste caso, ao término da soldagem a peça já se encontra com suas tensões aliviadas, não requerendo tratamento posterior. A Figura 4 mostra um condicionamento de solda sendo executado.

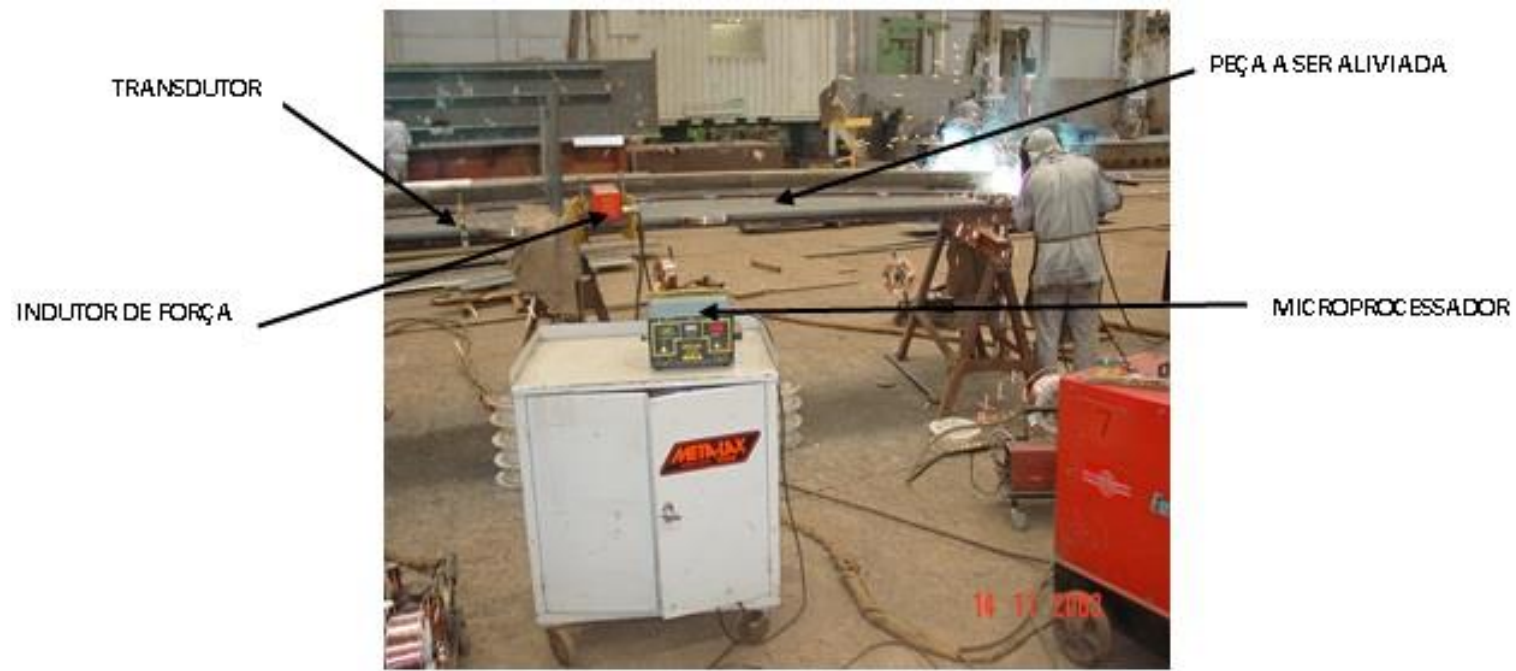

Figura 4: Condicionamento de solda em execução durante a soldagem. 


\section{MATERIAIS E MÉTODOS}

A Figura 5 mostra um desenho dos corpos de prova de aço ABNT 4140 forjado, temperado a chama e revenido, com dureza superficial de $58 \pm 2 \mathrm{HRC}$, nas medidas de 20,0 x $100 \times 70 \mathrm{~mm}$. As propriedades mecânicas do material foram ensaiadas conforme a ASTM A 370/12 com os seguintes resultados:

Limite de Resistência: $800 \mathrm{~N} / \mathrm{mm}^{2}$; Limite de Escoamento: $523 \mathrm{~N} / \mathrm{mm}^{2}$; Alongamento: 19\%.

Foram preparadas doze peças desse tipo, contendo tensões residuais provenientes dos processos de usinagem e têmpera, numeradas de 1 a 12.
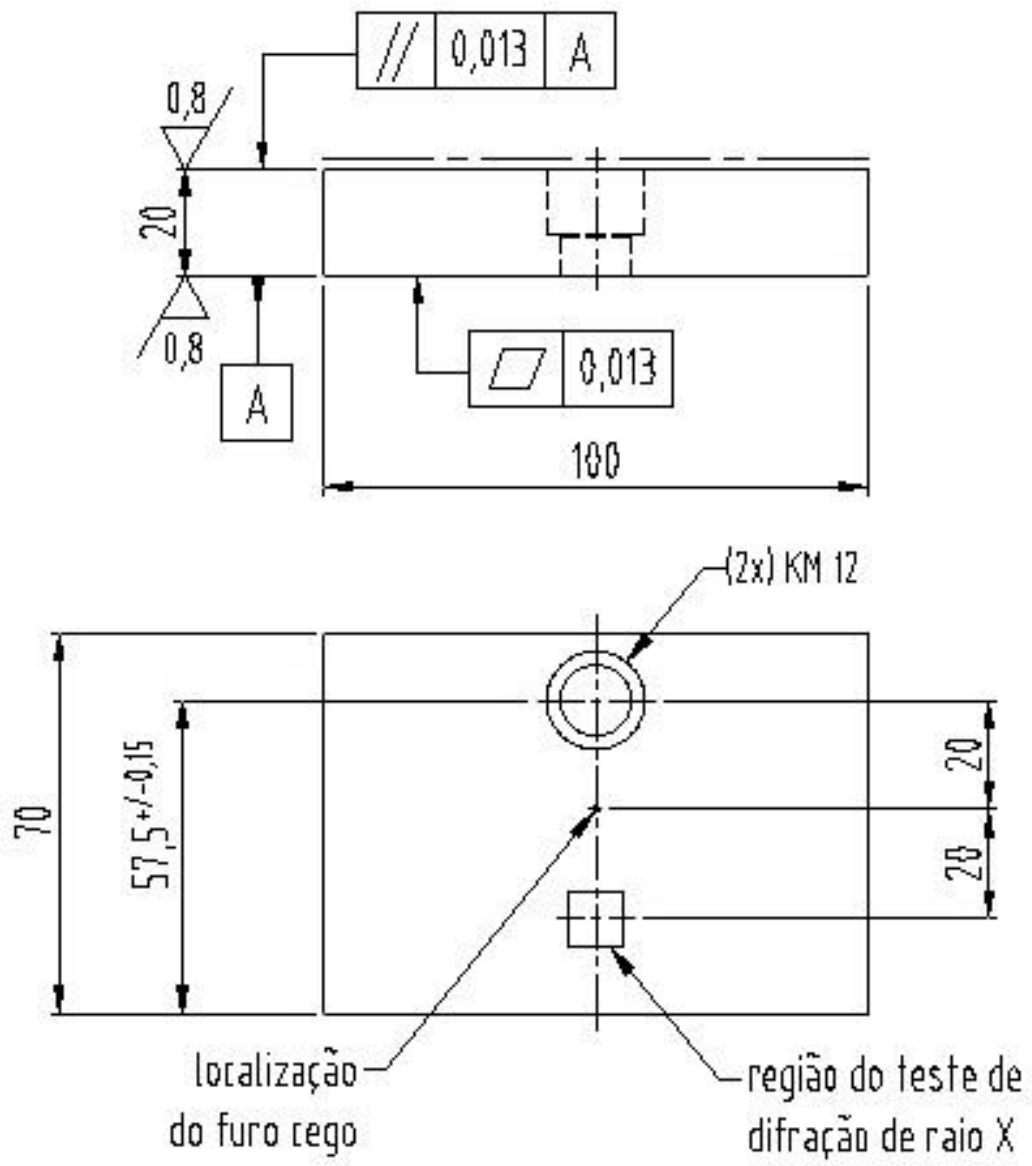

Figura 5: Desenho esquemático dos corpos de prova.

Os corpos de prova numerados de 1 a 6 tiveram as tensões residuais geradas por usinagem da superfície e os numerados de 7 a 12 tiveram as tensões introduzidas por têmpera. Essas peças foram então aliviadas por tratamentos térmico convencional e por vibrações sub-ressonantes. As tensões existentes foram medidas antes e após os tratamentos, com as técnicas de furo cego e por difração de raios X. Os corpos de prova de número par tiveram suas tensões aliviadas pelo método térmico e os corpos de número ímpar por vibrações.

A Tabela1 resume todas as condições experimentais usadas. 
Tabela 1: Relação dos tratamentos e testes executados nos corpos de prova.

\begin{tabular}{|c|c|c|c|c|c|c|}
\hline \multirow{2}{*}{$\begin{array}{l}\text { ATIVIDADE } \\
\text { CORPO DE } \\
\text { PROVA }\end{array}$} & \multicolumn{2}{|c|}{$\begin{array}{c}\text { INTRODUÇÃO DE } \\
\text { TENSÕES RESIDUAIS }\end{array}$} & \multicolumn{2}{|c|}{ ALÍVIO DE TENSÕES } & \multicolumn{2}{|c|}{$\begin{array}{c}\text { MEDIÇÃO DE TENSÕES } \\
\text { RESIDUAIS }\end{array}$} \\
\hline & USINAGEM & TÊMPERA & TÉRMICO & VIBRAÇÕES & $\begin{array}{c}\text { TESTE DE } \\
\text { FURO } \\
\text { CEGO } \\
\end{array}$ & $\begin{array}{l}\text { DIFRAÇÃO } \\
\text { DE RAIOS-X }\end{array}$ \\
\hline 1 & $\bullet$ & & & $\bullet$ & $\bullet$ & $\bullet$ \\
\hline 2 & $\bullet$ & & $\bullet$ & & $\bullet$ & $\bullet$ \\
\hline 3 & $\bullet$ & & & $\bullet$ & $\bullet$ & $\bullet$ \\
\hline 4 & $\bullet$ & & $\bullet$ & & $\bullet$ & $\bullet$ \\
\hline 5 & $\bullet$ & & & $\bullet$ & $\bullet$ & $\bullet$ \\
\hline 6 & $\bullet$ & & $\bullet$ & & $\bullet$ & $\bullet$ \\
\hline 7 & & $\bullet$ & & $\bullet$ & $\bullet$ & $\bullet$ \\
\hline 8 & & $\bullet$ & $\bullet$ & & $\bullet$ & $\bullet$ \\
\hline 9 & & $\bullet$ & & $\bullet$ & $\bullet$ & $\bullet$ \\
\hline 10 & & $\bullet$ & $\bullet$ & & $\bullet$ & $\bullet$ \\
\hline 11 & & $\bullet$ & & $\bullet$ & $\bullet$ & $\bullet$ \\
\hline 12 & & $\bullet$ & $\bullet$ & & $\bullet$ & $\bullet$ \\
\hline
\end{tabular}

\subsection{Usinagem dos Corpos de Prova}

Os corpos de prova primeiramente foram desbastados para corrigir desvios de paralelismo entre a mesa da fresadora e a superfície das peças. Para a operação de desbaste foram utilizadas ferramentas de corte modelo Coromil 245 M da empresa Sandvik, montadas em um cabeçote de $80 \mathrm{~mm}$ de diâmetro e utilizados os parâmetros mostrados na Tabela 2:

Tabela 2: Parâmetros utilizados na operação de desbaste

\begin{tabular}{l|c}
\hline Velocidade de corte $\left(\mathrm{v}_{\mathrm{c}}\right)$ & $277 \mathrm{~m} / \mathrm{min}$ \\
\hline Rotação $(\mathrm{n})$ & $700 \mathrm{rpm}$ \\
\hline Velocidade de avanço $\left(\mathrm{V}_{\mathrm{f}}\right)$ & $250 \mathrm{~mm} / \mathrm{min}$ \\
\hline Profundidade de corte $\left(\mathrm{a}_{\mathrm{p}}\right)$ & $0,1 \mathrm{~mm}$ \\
\hline Diâmetro da fresa & $80 \mathrm{~mm}$ \\
\hline Arestas de corte & 6 \\
\hline Refrigeração & Sem uso de refrigerante \\
\hline Ferramenta de corte & Coromill $245 \mathrm{M} /$ Sandvik \\
\hline
\end{tabular}

A usinagem de desbaste e de acabamento dos corpos de prova foi executada em uma fresadora Sanches Blanes, modelo FU-1 (ISO 40) com rotação máxima de $6.000 \mathrm{rpm}$, avanço de até $1.500 \mathrm{~mm} / \mathrm{min}$ e painel de operação digital para os eixos X, Y e Z.

Para as operações de acabamento foram utilizados insertos de CBN código R245 12T3 E CB50 de duas arestas cortantes, montados em um cabeçote de $63 \mathrm{~mm}$ de diâmetro da empresa Sandvik, sem utilização de fluido refrigerante ou de corte. Os parâmetros de usinagem estão descritos na Tabela 3. 
Tabela 3: Parâmetros de usinagem utilizados.

\begin{tabular}{c|c|c|c|c|c}
\hline \multirow{2}{*}{$\begin{array}{c}\text { CORPO } \\
\text { DE }\end{array}$} & $\begin{array}{c}\text { VELOCIDADE } \\
\text { PROVA }\end{array}$ & AVANÇO & $\begin{array}{c}\text { PROFUNDIDADE } \\
\text { PENETRAÇÃO }\end{array}$ & ROTAÇÃO & $\begin{array}{c}\text { VELOCIDADE } \\
\text { DE AVANÇO }\end{array}$ \\
\cline { 2 - 6 } & $\mathrm{V}_{\mathrm{c}}(\mathrm{m} / \mathrm{min})$ & $\mathrm{f}_{2}(\mathrm{~mm} / \mathrm{rev})$ & $\mathrm{a}_{\mathrm{p}}(\mathrm{mm})$ & $\mathrm{n}(\mathrm{rpm})$ & $\mathrm{V}_{\mathrm{f}}(\mathrm{mm} / \mathrm{min})$ \\
\hline $\mathbf{1}$ & 351 & 0,100 & 0,15 & 1774 & 867,0 \\
\hline $\mathbf{2}$ & 225 & 0,100 & 0,15 & 1137 & 568,4 \\
\hline $\mathbf{3}$ & 225 & 0,100 & 0,07 & 1137 & 568,4 \\
\hline $\mathbf{4}$ & 225 & 0,100 & 0,23 & 1137 & 568,4 \\
\hline $\mathbf{5}$ & 300 & 0,050 & 0,10 & 1516 & 378,9 \\
\hline $\mathbf{6}$ & 300 & 0,150 & 0,20 & 1516 & 1136,8 \\
\hline
\end{tabular}

As alterações da velocidade de corte, profundidade e penetração e da velocidade de avanço foram usadas para produzir variações nas tensões residuais dos corpos de prova, para melhor avaliação dos dois processos de alívio, conforme demonstrado por CHEVRIER [9] e GUNNBERG et al. [10].

\subsection{Têmpera dos Corpos de Prova}

Os seis corpos de prova foram temperados à temperatura de $920^{\circ} \mathrm{C}$, encharque de 40 minutos e com os seguintes resfriamentos: em óleo sem agitação (CP's 7 e 8); em salmoura sem agitação (CP's 9 e 10); com fluxo de ar forçado (CP's 11 e 12), de forma a se ter ciclos térmicos com crescente severidade e diferentes níveis de tensões residuais.

\subsection{Medições das Tensões Residuais Antes e Após os Alívios}

As tensões residuais foram medidas pelo método do furo cego desde a superfície até a profundidade de 0,4 a 0,6 mm, nos dois lados da peça e por difração de raios-X somente na superfície, utilizando-se o método de $\operatorname{sen}^{2} \psi$. A área da superfície analisada foi de $10 \mathrm{~mm}^{2}$, na região central e na direção transversal à peça, sendo utilizados os ângulos de incidência $\Psi$ iguais a 15,30 e 45 , modo de varredura contínua com velocidade de 2 graus/minuto, tempo de exposição 1,5 segundos, com radiação de $40 \mathrm{kV}$ e $30 \mathrm{~mA}$. Os locais de medição das duas técnicas estão ilustrados na figura 5 e receberam polimento eletrolítico, composto de $800 \mathrm{ml}$ de etanol absoluto e $60 \mathrm{ml}$ de $\mathrm{HClO}_{4}(60 \%)$.

Para a técnica do furo cego, foi utilizado o equipamento ilustrado na Figura 6a, composto de uma fresadora Hottinger Baldwin, modelo Messtechinik, de acionamento pneumático e fixação magnética, velocidade máxima de $400.000 \mathrm{rpm}$, com broca de 2,2 mm, acoplada a um sistema automático de medição de tensões residuais Sint Technology, cujos dados foram registrados em um computador com o software H-Drill. A Figura 6 b é um esquema do equipamento usado.

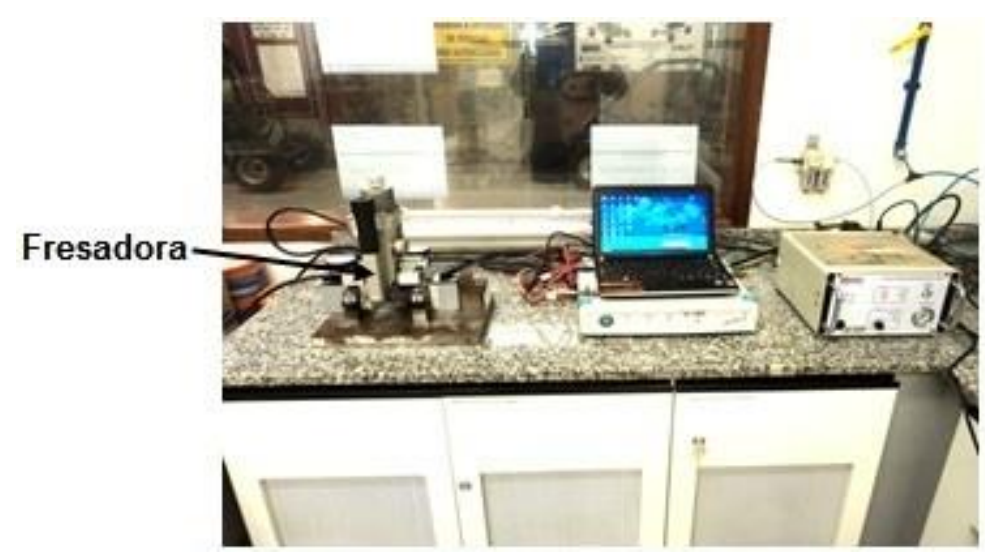

Figura 6a: Equipamento para medição de tensões (furo cego). 


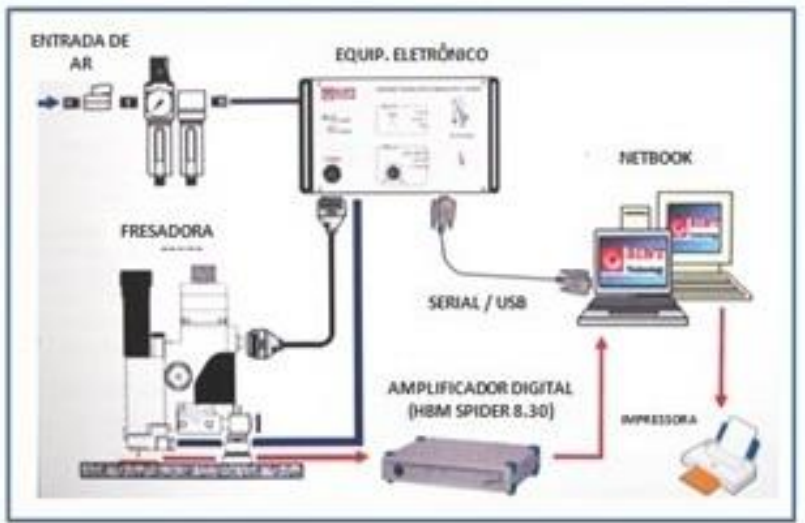

Figura 6b: Representação esquemática do equipamento

Para a medição de tensões residuais por difração de raios-X, foi utilizado o equipamento Shimadzu modelo XRD-7000, mostrado na Figura 7.

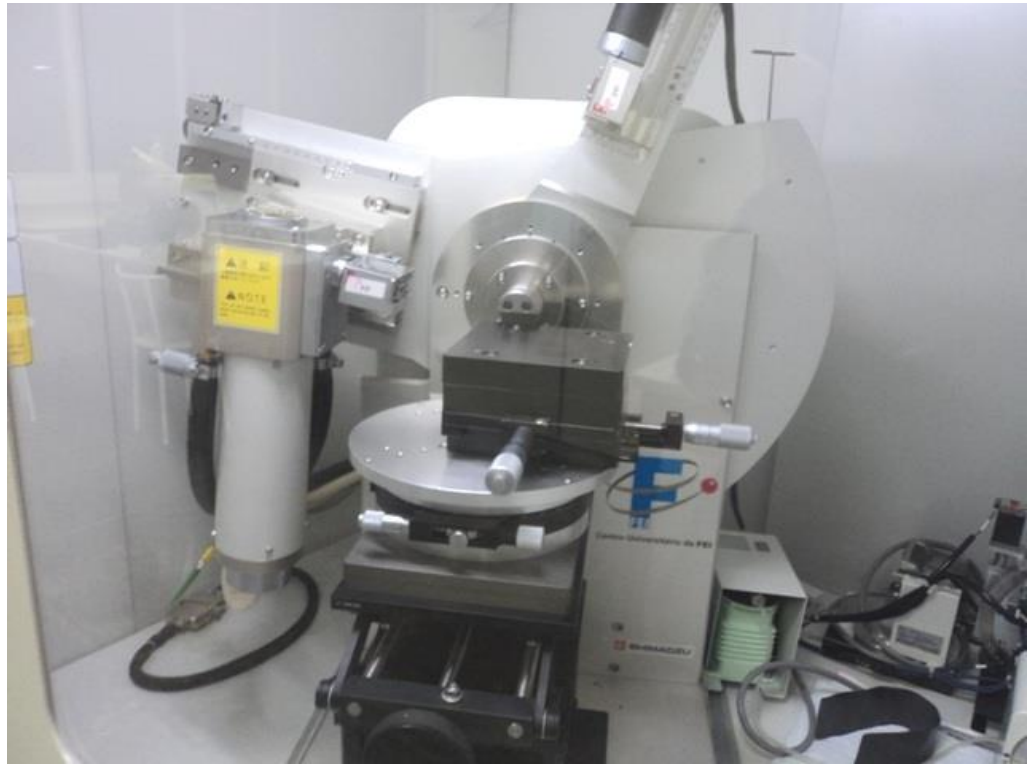

Figura 7: Equipamento de difração de Raios-X.

\subsection{Alívio de Tensões por Vibrações Sub-ressonantes}

Os corpos de prova 1, 3, 5, 7, 9 e 11 foram aliviados através da técnica de vibrações sub-ressonantes com a utilização do equipamento Meta-lax Série 2700 automático, utilizando um indutor de força 2A, com ajuste de excêntricos a $40 \%$. As massas dos corpos de prova são baixas em relação à capacidade do equipamento, por este motivo foram aliviados aos pares, fixados sobre a mesa oscilante. Para o alívio, foi utilizada a frequência de $70 \mathrm{~Hz}$. O tempo de aplicação das vibrações foi inicialmente de 20 minutos e de 10 minutos nas aplicações posteriores.

\subsection{Alívio de Tensões por Tratamento Térmico}

Os corpos de prova 2, 4, 6, 8, 10 e 12 foram tratados termicamente de acordo com a norma AWS D1.1 (2004), que especifica temperatura entre $600^{\circ} \mathrm{C}$ e $650^{\circ} \mathrm{C}$ por um período de 2 horas para materiais com espessura até 2 polegadas $(51 \mathrm{~mm})$. Neste procedimento foi utilizado um forno marca Jung modelo 1514 de $3,1 \mathrm{KW}$.

\section{RESULTADOS E DISCUSSÃO}

Os resultados dos ensaios de furo cego, antes e após os alívios, estão apresentados na Tabela 4, cujas tensões foram observadas a profundidade de $0,2 \mathrm{~mm}$, no eixo x e no eixo de laminação y, mostrados na Figura 8 . A profundidade de $0,2 \mathrm{~mm}$ foi utilizada por evidenciar a faixa onde são concentradas as maiores tensões residu- 
ais internas, quando analisados os gráficos dos referidos testes.

Tabela 4: Tensões residuais obtidas nos ensaios de furo cego antes e após os alívios na profundidade de 0,2 mm

\begin{tabular}{|c|c|c|c|c|c|c|}
\hline \multirow{2}{*}{$\begin{array}{c}\text { CORPO } \\
\text { DE PRO- } \\
\text { VA }\end{array}$} & \multirow{2}{*}{$\begin{array}{c}\text { GERAÇÃO DE } \\
\text { TENSÕES RESI- } \\
\text { DUAIS }\end{array}$} & \multirow{2}{*}{$\begin{array}{l}\text { ALÍVIO DE } \\
\text { TENSÕES }\end{array}$} & \multicolumn{2}{|c|}{$\begin{array}{c}\text { TENSÕES ANTES DO } \\
\text { ALÍVIO }\end{array}$} & \multicolumn{2}{|c|}{ TENSÕES APÓS O ALÍVIO } \\
\hline & & & $\begin{array}{l}\text { EIXO X } \\
\text { (MPA) }\end{array}$ & $\begin{array}{l}\text { EIXO Y } \\
\text { (MPA) }\end{array}$ & $\begin{array}{l}\text { EIXO X } \\
\text { (MPA) }\end{array}$ & $\begin{array}{l}\text { EIXO Y } \\
\text { (MPA) }\end{array}$ \\
\hline CP-1 & Usinagem & Vibrações & -750 & -1000 & 0 & 0 \\
\hline $\mathrm{CP}-2$ & Usinagem & Térmico & -750 & -1200 & -500 & -500 \\
\hline CP-3 & Usinagem & Vibrações & -750 & -750 & 0 & 0 \\
\hline CP-4 & Usinagem & Térmico & -750 & -1100 & 0 & 0 \\
\hline $\mathrm{CP}-5$ & Usinagem & Vibrações & -600 & -350 & +30 & +20 \\
\hline CP-6 & Usinagem & Térmico & -700 & -1000 & +100 & +180 \\
\hline $\mathrm{CP}-7$ & Têmpera & Vibrações & +500 & +600 & -150 & -150 \\
\hline CP-8 & Têmpera & Térmico & +550 & +600 & +250 & +300 \\
\hline CP-9 & Têmpera & Vibrações & -150 & -300 & -120 & -160 \\
\hline CP-10 & Têmpera & Térmico & -150 & -290 & -150 & -170 \\
\hline CP-11 & Têmpera & Vibrações & +110 & +130 & 0 & 0 \\
\hline CP-12 & Têmpera & Térmico & +140 & +160 & +60 & +30 \\
\hline
\end{tabular}

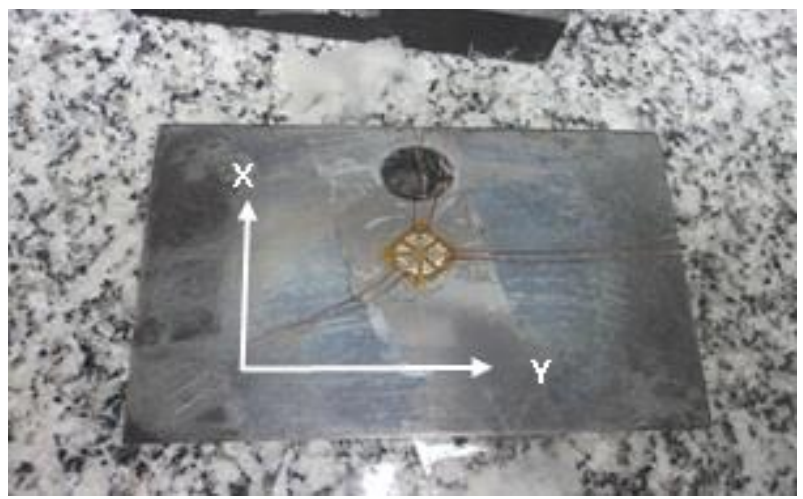

Figura 8: Localização dos eixos x e y.

Nos testes de furo cego observou-se que os níveis de tensões residuais encontrados após os alívios por vibrações sub-ressonantes e térmicos são equivalentes a partir da profundidade de $0,2 \mathrm{~mm}$ e que as tensões residuais tenderam a zero após os alívios.

Os resultados pontuais obtidos dos testes de difração de raios-X, analisados na região mostrada na Figura 5, estão apresentados na Tabela 5.

Tabela 5: Tensões residuais obtidas por difração de raios-X, antes e após os alívios.

\begin{tabular}{|c|c|c|c|c|c|c|}
\hline \multirow{2}{*}{$\begin{array}{l}\text { CORPO } \\
\text { DE } \\
\text { PROVA }\end{array}$} & \multirow{2}{*}{$\begin{array}{c}\text { INTRODUÇÃO } \\
\text { DE TENSÕES } \\
\text { RESIDUAIS }\end{array}$} & \multirow{2}{*}{$\begin{array}{l}\text { ALÍVIO DE } \\
\text { TENSÕES }\end{array}$} & \multicolumn{4}{|c|}{$\begin{array}{c}\text { TENSÕES ANALISADAS POR DIFRAÇÃO DE RAI- } \\
\text { OS-X }\end{array}$} \\
\hline & & & ANTES (MPA) & $\begin{array}{l}\text { DESVIO } \\
\text { (MPA) }\end{array}$ & $\begin{array}{l}\text { APÓS } \\
\text { (MPA) }\end{array}$ & $\begin{array}{l}\text { DESVIO } \\
\text { (MPA) }\end{array}$ \\
\hline CP-1 & Usinagem & Vibrações & -368 & 20 & +110 & 6 \\
\hline CP-2 & Usinagem & Térmico & -298 & 22 & -303 & 28 \\
\hline $\mathrm{CP}-3$ & Usinagem & Vibrações & -258 & 24 & -297 & 13 \\
\hline $\mathrm{CP}-4$ & Usinagem & Térmico & -320 & 34 & -321 & 10 \\
\hline $\mathrm{CP}-5$ & Usinagem & Vibrações & -403 & 16 & +171 & 24 \\
\hline CP-6 & Usinagem & Térmico & -225 & 45 & -363 & 16 \\
\hline CP-7 & Têmpera & Vibrações & -79 & 4 & +7 & 1 \\
\hline CP-8 & Têmpera & Térmico & -79 & 4 & -389 & 11 \\
\hline CP-9 & Têmpera & Vibrações & -84 & 0 & +100 & 16 \\
\hline CP-10 & Têmpera & Térmico & -84 & 0 & -349 & 15 \\
\hline
\end{tabular}




\begin{tabular}{|c|c|c|c|c|c|c|}
\hline \multirow{2}{*}{$\begin{array}{c}\text { CORPO } \\
\text { DE } \\
\text { PROVA }\end{array}$} & \multirow{2}{*}{$\begin{array}{l}\text { INTRODUÇÃO } \\
\text { DE TENSOOES } \\
\text { RESIDUAIS }\end{array}$} & \multirow{2}{*}{$\begin{array}{l}\text { ALÍVIO DE } \\
\text { TENSÕES }\end{array}$} & \multicolumn{4}{|c|}{$\begin{array}{l}\text { TENSÕES ANALISADAS POR DIFRAÇÃO DE RAI- } \\
\text { OS-X }\end{array}$} \\
\hline & & & ANTES (MPA) & $\begin{array}{l}\text { DESVIO } \\
\text { (MPA) }\end{array}$ & $\begin{array}{l}\text { APÓS } \\
\text { (MPA) }\end{array}$ & $\begin{array}{l}\text { DESVIO } \\
\text { (MPA) }\end{array}$ \\
\hline $\mathrm{CP}-11$ & Têmpera & Vibrações & -77 & 3 & +412 & 47 \\
\hline CP-12 & Têmpera & Térmico & -77 & 3 & -271 & 11 \\
\hline
\end{tabular}

Pode ser observado que o processo de usinagem introduz tensões residuais maiores que as induzidas por têmpera. Na superfície, elas são em média 390\% maiores. Abaixo da superfície os valores absolutos das tensões são maiores que os medidos na superfície. Esta variação é explicada pela falta de concordância que existe entre as duas técnicas quando se utiliza brocas maiores que $0,8 \mathrm{~mm}$ de diâmetro para o teste de furo cego, conforme comprovado por ROCHA [11]. Pelo mesmo motivo, também se observa que o alívio não ocorre de forma similar na superfície e no interior da peça.

Os resultados obtidos com ambos os métodos de medição indicaram que ocorreram casos de inversão das tensões residuais, de compressão (negativas) para tensões de tração (positivas) após tratamento, mas isso ocorreu principalmente na superfície. Isso pode ser explicado pela teoria do deslocamento estendido, citada por KUO et al. [12], e pelo modelo de plasticidade para a operação de alívio de tensões por vibração apresentado por WALKER [13].

Comparando-se as médias das variações de tensão antes e após o alívio, para os dois métodos de geração de tensões residuais e para os dois métodos de alívio, os resultados estão ilustrados na Tabela 6.

Tabela 6: Comparação entre as médias das variações de tensões residuais pelos métodos de geração e alívio, antes e após os tratamentos.

\begin{tabular}{c|c|c|c}
\hline \multirow{4}{*}{ GERAÇÃo } & $\begin{array}{c}\text { ALÍVIO DE } \\
\text { TENSÕES }\end{array}$ & MEDIÇÃo & $\begin{array}{c}\text { MÉDIAS DAS VARIAÇÕES } \\
\text { DE TENSÕES RESIDUAIS } \\
\text { (MPA) }\end{array}$ \\
\hline \multirow{4}{*}{ Usinagem } & Térmico & Difração & 48,0 \\
\cline { 2 - 4 } & Térmico & Furo Cego & 796,7 \\
\cline { 2 - 4 } & Vibrações & Difração & 365,7 \\
\cline { 2 - 4 } & Vibrações & Furo Cego & 708,3 \\
\hline \multirow{4}{*}{ Têmpera } & Térmico & Difração & 256,3 \\
\cline { 2 - 4 } & Térmico & Furo Cego & 268,3 \\
\cline { 2 - 4 } & Vibrações & Difração & 253,0 \\
\cline { 2 - 4 } & Vibrações & Furo Cego & 306,7 \\
\hline
\end{tabular}

Os resultados obtidos na Tabela 6 mostram que a média das variações de tensões residuais analisadas através do método de furo cego são $125,4 \%$ maiores que as analisadas através da difração por raios-x, evidenciando a falta de concordância entre as técnicas de medição apresentada por ROCHA [11]. Também pode ser observado que o nível de remoção de tensões pelo alívio por vibrações é 19,3\% maior que pelo alívio térmico,

Por meio dos resultados ilustrados na Tabela 4 e os obtidos dos relatórios dos alívios de tensões por vibrações permitem correlacionar os valores das tensões removidas com as diferenças das frequências entre os picos de ressonância encontrados antes e após a execução do alívio de tensões. A Tabela 7 apresenta esta correlação. 
Tabela 7: Correlação entre as tensões residuais removidas e a diferença de frequência entre os picos de ressonância

\begin{tabular}{|c|c|c|c|c|c|}
\hline \multirow{2}{*}{$\begin{array}{l}\text { CORPO DE } \\
\text { PROVA }\end{array}$} & \multirow{2}{*}{$\begin{array}{c}\text { GERAÇÃO DE } \\
\text { TENSÕES RESI- } \\
\text { DUAIS }\end{array}$} & \multirow{2}{*}{$\begin{array}{l}\text { ALÍVIO } \\
\text { DE TEN- } \\
\text { SÕES }\end{array}$} & \multicolumn{2}{|c|}{$\begin{array}{c}\text { DIFERENÇA EM MÓDULO } \\
\text { DAS TENSÕES ANTES E } \\
\text { APÓS O ALÍVIO }(\Delta \sigma)\end{array}$} & \multirow{2}{*}{$\begin{array}{c}\text { FREQUENCIA ENTRE } \\
\text { PICOS DE RESSO- } \\
\text { NÂNCIA } \\
(\Delta \mathrm{f}) \\
\text { DIFERENÇA } \\
(\mathrm{HZ})\end{array}$} \\
\hline & & & $\begin{array}{c}\text { EIXO X } \\
\text { (MPA) }\end{array}$ & $\begin{array}{l}\text { EIXO Y } \\
\text { (MPA) }\end{array}$ & \\
\hline $\mathrm{CP}-1$ & Usinagem & Vibrações & 750 & 1000 & 1,0 \\
\hline $\mathrm{CP}-3$ & Usinagem & Vibrações & 750 & 750 & 1,0 \\
\hline CP-5 & Usinagem & Vibrações & 630 & 370 & 0,7 \\
\hline CP-7 & Têmpera & Vibrações & 650 & 750 & 0,7 \\
\hline CP-9 & Têmpera & Vibrações & 30 & 140 & 0,4 \\
\hline CP-11 & Têmpera & Vibrações & 110 & 130 & 0,4 \\
\hline
\end{tabular}

A redução das tensões obtida nos tratamentos térmico e por vibrações sub-ressonantes não é uniforme na superfície dos materiais. Este fato já havia sido evidenciado em pesquisas anteriores efetuadas por WALKER (1995); [14]

Levando-se em consideração os dados apresentados na Tabela 6 e utilizando-se estudos do melhor ajuste de curvas sobre as séries, foram obtidos os seguintes resultados de coeficientes de correlação nas respectivas equações: linear $\left(\mathrm{R}^{2}=0,9247\right)$; exponencial $\left(\mathrm{R}^{2}=0,913\right)$; logarítmica $\left(\mathrm{R}^{2}=0,982\right)$; polinomial $\left(\mathrm{R}^{2}=\right.$ $0,986)$ e função potência $\left(R^{2}=0,988\right)$. Deste modo, foi determinada a equação que permite estimar o valor das tensões residuais aliviadas a partir da diferença de frequência entre os picos de ressonância, antes e após o alívio para os eixos $\mathrm{x}$ e $\mathrm{y}$.

Para os eixos x e y, foi determinada a equação abaixo, com coeficiente de correlação $\mathrm{R}^{2}=0,988$ :

$$
\Delta \sigma=196,6(\Delta \mathrm{f})^{0,821}
$$

Substituindo as diferenças de frequência $(\Delta f)$ na equação 1 , são encontrados os valores correspondentes às tensões residuais removidas da peça $\Delta \sigma(\mathrm{MPa})$. Estes valores estão apresentados na figura 9 , que mostra esta relação.

Para os corpos de prova CP-1 e CP-3, demonstrados na tabela 7, a diferença de frequências foi de 1,0 HZ. A figura 9 apresenta o gráfico de frequências para estes corpos.

\begin{tabular}{|c|c|}
\hline \multicolumn{2}{|c|}{ Equação $1 ; R^{2}=0,988$} \\
\hline$\Delta \mathrm{f}(\mathrm{Hz})$ & $\Delta \sigma(\mathrm{MPa})$ \\
\hline 0,5 & 111 \\
\hline 1,0 & 197 \\
\hline 1,5 & 274 \\
\hline 2,0 & 347 \\
\hline 2,5 & 417 \\
\hline 3,0 & 485 \\
\hline 3,5 & 550 \\
\hline 4,0 & 614 \\
\hline 4,5 & 676 \\
\hline 5,0 & 737 \\
\hline 5,5 & 797 \\
\hline 6,0 & 856 \\
\hline 6,5 & 914 \\
\hline 7,0 & 971 \\
\hline 7,5 & 1.028 \\
\hline 8,0 & 1.084 \\
\hline 8,5 & 1.139 \\
\hline 9,0 & 1.194 \\
\hline 9,5 & 1.248 \\
\hline 10,0 & 1.302 \\
\hline
\end{tabular}

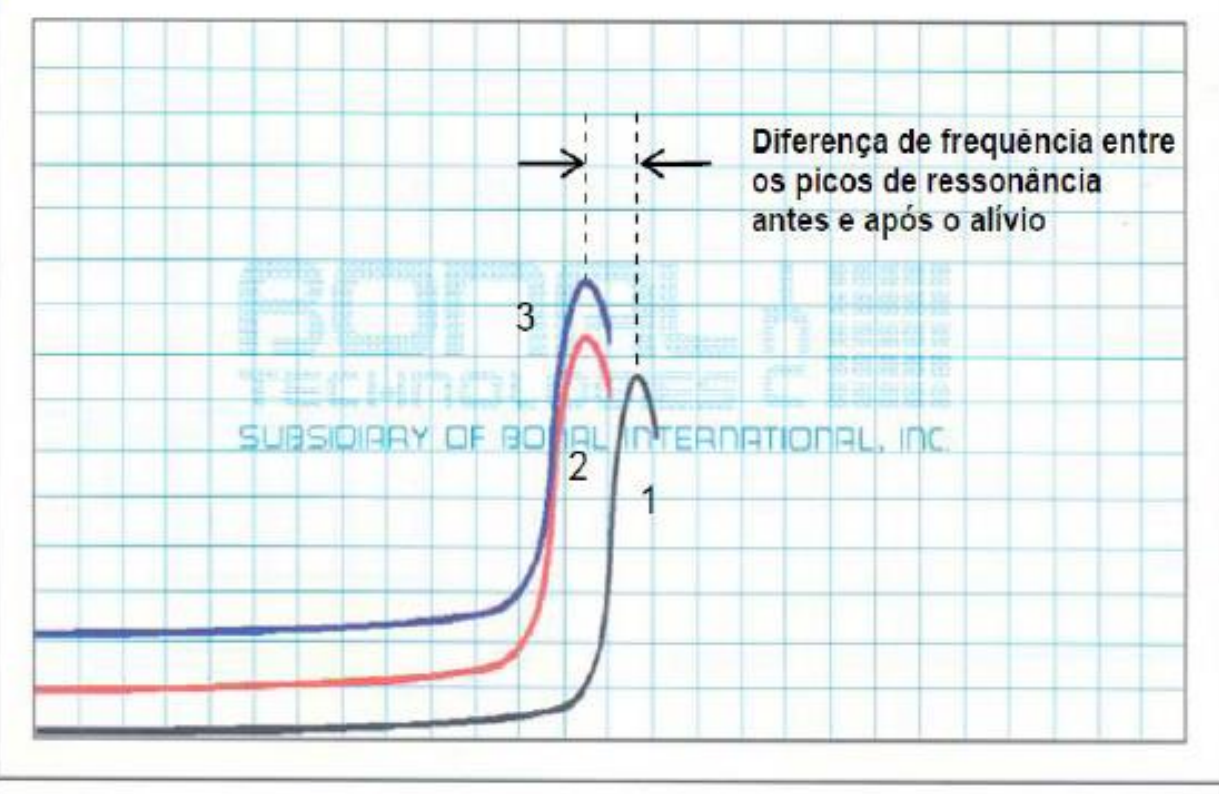

Figura 9: Diferença de frequência $\Delta \mathrm{f}(\mathrm{Hz}) \mathrm{x}$ tensões residuais aliviadas $\Delta \sigma(\mathrm{MPa})$ 
No gráfico podem ser observadas três curvas. A curva 1 é a obtida no estado original de tensões da peça. A curva 2 é a curva obtida após o primeiro alívio. Note que a diferença entre as frequências de ressonância é de $1 \mathrm{~Hz}$. A curva 3 é a curva obtida após o segundo alívio. A frequência de ressonância dessa última curva é a mesma que a da segunda, indicando que não existem mais tensões passíveis de remoção.

Observando que a diferença entre as frequências de ressonância das curvas 1 e 3 é de $1 \mathrm{~Hz}$ e considerando a equação 1, obtém-se uma redução de tensão de 197 MPa.

Feito o mesmo estudo para os corpos de prova 5 e 7 e os 9 e 11, as remoções de tensões foram de 147 $\mathrm{MPa}$ e de $93 \mathrm{MPa}$, respectivamente.

\section{CONCLUSÕES}

Neste artigo evidenciou-se através das medições que na profundidade de $0,2 \mathrm{~mm}$, faixa onde são concentradas as maiores tensões residuais internas, o tratamento térmico de alívio de tensões provocou reduções de tensão de até $100 \%$. Na mesma profundidade, o alívio de tensões por vibrações sub-ressonantes provoca reduções entre $20,0 \%$ e $100 \%$ das tensões residuais.

De forma mais geral, o alívio de tensões por vibrações sub-ressonantes na profundidade de 0,2mm, promove alívio de tensões percentuais médios em média $10 \%$ maiores, quando comparados com os resultados obtidos através do tratamento térmico. A média dos níveis de remoção de tensões pelo alívio por vibrações é $19,3 \%$ maior que pelo alívio térmico.

Os dados apresentados na tabela 2 , oriundos da equação 1 , correlacionam as tensões residuais removidas da peça, em função da diferença entres as frequências de ressonância antes e após o alívio, possibilitando visualizar os resultados obtidos com a aplicação do alívio de tensões por vibração.

Como consideração final, este trabalho mostra que, apesar de as instituições normativas não incluírem o tratamento por vibrações sub-ressonantes em seu elenco de técnicas para alívio de tensões, os resultados mostram que existe esta possibilidade. Fica patente a necessidade de realização de novas pesquisas a fim de se obter mais resultados qualitativos e quantitativos, inclusive para tensões residuais originadas por outros processos de fabricação/tratamento, particularmente soldagem.

\section{AGRADECIMENTOS}

Os autores agradecem à ENGEBASA - Engebasa Mecânica e Usinagem Ltda. e seus diretores pela colaboração prestada e informações cedidas, que possibilitaram a preparação desse trabalho.

\section{BIBLIOGRAFIA}

[1] MARQUES, P. V., MARTINS, C. A. P., "Alívio de Tensões e Condicionamento de Solda por Vibrações Sub-Ressonantes”, In: XXXIV Congresso Nacional de Soldagem ABS, São Paulo, São Paulo, 2008.

[2] MODENESI, P. J., MARQUES, P. V., SANTOS, D. B. “Introdução a Metalurgia da Soldagem”, Universidade Federal de Minas Gerais, Belo Horizonte, 2012. 209 p. Apostila. Disponível em: <http://demet.eng.ufmg.br/wp-content/uploads/2012/10/metalurgia.pdf>. Acesso em: 02 mar. 2016.

[3] MODENESI, P. J. “Efeitos Mecânicos do Ciclo Térmico”, Universidade Federal de Minas Gerais, Pampulha, 2008. 25 p. Apostila. Disponível em:

<http://www.iem.unifei.edu.br/professores/edmilson/tensaoresidual.pdf>. Acesso em: 15 mar. 2013.

[4] HEBEL, Thomas E. "Heat Treating, Stress relieving”, In: Metal Casting, pp.1-4. Sep 1989.

[5] SKINNER, R. D. An investigation Into the Theory Behind Sub-resonant Stress Relieve, In: Lockheed Missiles and Aerospace Report, USA, 1987.

[6] WONG, T. E., JOHNSON, G. C. "Ultrasonic Evaluation of the Nonlinearity of Metals from a Design Perspective”, Department of Mechanical Engineering, University of California, Berkeley, USA, 1987.

[7] MARTINS, C. A. P., MORILLA, J.C., MARQUES, P. V., et al., "Alívio de Tensões e Condicionamento de Solda por Vibrações Sub-Ressonantes", In: XXXVIII Congresso Nacional de Soldagem ABS, Ouro Preto, Minas Gerais, 2012.

[8] BONAL TECHNOLOGIES, INC. http://www.meta-lax.com/Home/Benefits/benefits.html. Acessado em: 24de março de 2013.

[9] CHEVRIER, P., TIDU, A., BOLLE, B., et al., "Investigation of surface integrity in high speed end milling of a low alloyed steel”, International Journal of Machine Tools \& Manufacture, v.43, pp.1135-1142, 2003. 
[10] GUNNBERG, F., ESCURSELL, M., JACOBSON, M., et al., "The influence of cutting parameters on residual stresses and surface topography during hard turning of $18 \mathrm{MnCr} 5$ case carburized steel", Journal of Materials Processing Technology, v.174, pp.82-90, 2006.

[11] ROCHA, A.S., NUNES, R.M., HIRSCH, T.K. “Comparação entre Difração de Raio X e Método do Furo Cego para Medição de Tensões Residuais em Barras Cilíndricas”, Revista Matéria, v. 14, n. 3, pp. 965 976, Rio de Janeiro, 2009.

[12] KUO, C. W., LIN, C. M., LAI, G. H, et al., "Characterization and Mechanism of 304 Stainless Steel Vibration Welding”. Materials Transactions, v. 48, n. 9, pp. 2319-2323, Japan 2007.

[13] WALKER, C. "A Theoretical Review of the Operation of Vibratory Stress Relief”. Journal of Materials Design and Applications, pp. 225-125, Glasgow, UK, Jun. 2011.

[14] WALKER, C. A., WADDEL A. J., JOHNSTON D. J. "Vibratory Stress Relief - An Investigation of the Underlying Process - Alívio de Tensões por Vibrações - Uma Investigação do Processo Fundamental”, Journal of Process Mechanical Engineering Periódico de Engenharia de Processo Mecânico, v. 209, pp. 5158, Fev. 1995. 